\section{Optimal Inversion-Based Control for the Set-Point Regulation of Nonminimum-Phase Uncertain Scalar Systems}

\author{
Aurelio Piazzi and Antonio Visioli
}

\begin{abstract}
In this note, 1 we propose an optimal inversion-based control for the set-point constrained regulation of nonminimum-phase uncertain scalar systems. This approach is based on a new control architecture where the main purpose of the feedback is to reduce the sensitivity to parametric plant uncertainties permitting in such a way the effective use of a feedforward action determined via a stable dynamic inversion. Essential constituents of the architecture are a parameterized family of "transition" polynomials to shape ideal output transfers and a parameterized controller ensuring the internal model principle. The methodology is then centered on the optimal combined design of the feedback controller and of the inversion-based command signal in order to minimize the worst-case settling time subject to an amplitude constraint on the control variable and to arbitrarily assigned overshoot and undershoot bounds. Finally, an approximate or suboptimal solution to the resulting nonlinear optimization problem can be obtained with genetic algorithms. A worked example highlights the effectiveness of the overall methodology.
\end{abstract}

Index Terms-Inversion-based control, nonminimum-phase systems, optimization, robust performance, set-point regulation.

\section{INTRODUCTION}

In this note, we focus our efforts on an optimal inversion-based synthesis of a feedforward/feedback strategy for the set-point constrained regulation of nonminimum-phase scalar systems nonlinearly depending on uncertain parameters. Specifically, the addressed problem is the determination of a command signal and of a feedback controller that minimize the worst-case settling time subject to arbitrarily assigned overshoot and undershoot constraints and to an amplitude limit for the plant control input. The proposed solution is based on a new control architecture where the main purpose of the feedback is to reduce the sensitivity to parametric plant uncertainties permitting in such a way the effective use of a feedforward action determined via a stable dynamic inversion. Essential constituents of the architecture are a parameterized family of "transition" polynomials [1] to shape ideal output transfers and a parameterized controller ensuring the internal model principle. The methodology is finally centered on the optimal combined design of the feedback controller and of the inversion-based command signal in order to minimize the worst-case settling time subject to all the required constraints.

It is worth noting that the proposed control architecture that generalizes for nonminimum-phase plants a previous approach reported in [2] is a novelty with respect to the inversion-based control scheme well-known in the technical literature [3], [4]. Indeed, as also reported by Devasia in [5] specifically addressing plant uncertainty in the inversion-based output tracking, stable dynamic inversion is applied over the nominal plant and if initial condition mismatches, modeling errors,

Manuscript received November 6, 2000; revised February 9, 2001 and April 16, 2001. Recommended by Associate Editor A. Datta. This work was supported in part by MURST scientific research funds.

A. Piazzi is with the Dipartimento di Ingegneria dell'Informazione, University of Parma, Parco Area delle Scienze, 181A-I-43100 Parma, Italy (e-mail aurelio@ce.unipr.it).

A. Visioli is with the Dipartimento di Elettronica per l'Automazione, University of Brescia, Italy (e-mail: visioli@bsing.ing.unibs.it).

Publisher Item Identifier S 0018-9286(01)09541-1.

${ }^{1}$ This article is a revised version of a paper originally presented at the IEEE International Conference on Decision and Control, Sydney, 2000. and disturbances appears then the feedback control intervenes to reduce performance degradation. With this approach feedback design is independent from (inversion-based) feedforward design. Differently, with our approach the stable dynamic inversion is applied on the nominal closed-loop system, and the feedback design, although it could be independently performed from the command signal design (see [1, ex. 5.2]), it is better accomplished with an integrated feedforward/feedback design permitting to exploit an optimality criterion (see Section IV).

Section II poses the set-point constrained regulation problem within a general framework. The new inversion-based control architecture is exposed in Section III. First, the controller structure is chosen and the ideal output functions are succinctly presented [1]. Then, Section III-A faces the crucial synthesis of a bounded command signal via dynamic inversion. The pertinent stable inversion problem is posed and a new solution using Laplace transform techniques is offered (Theorem 1). Section IV presents a nonlinear optimization problem whose approximate solution, gained with genetic algorithms, ends the design procedure.

\section{The SET-Point Constrained REgulation Problem}

In the context of linear, time-invariant, continuous-time systems, consider an uncertain scalar strictly proper nonminimum-phase plant whose transfer function is:

$$
P(s ; \mathbf{q})=\frac{b(s ; \mathbf{q}) c(s ; \mathbf{q})}{s^{h} a(s ; \mathbf{q})}, \quad h \in\{0,1\}
$$

where

$$
\begin{array}{ll}
a(s ; \mathbf{q}) & =\sum_{i=0}^{n-h} a_{i}(\mathbf{q}) s^{i}, \quad b(s ; \mathbf{q})=\sum_{i=0}^{p} b_{i}(\mathbf{q}) s^{i} \\
c(s ; \mathbf{q}) & =\sum_{i=0}^{m} c_{i}(\mathbf{q}) s^{i}
\end{array}
$$

and $\mathbf{q}=\left[q_{1}, \ldots, q_{g}\right]^{\mathrm{T}}$ belongs to a given multidimensional interval (box) $Q=\left[q_{1}^{-}, q_{1}^{+}\right] \times \cdots \times\left[q_{g}^{-}, q_{g}^{+}\right]$. The order of the plant is $n$, its relative order is $\rho=n-m-p$. Polynomials $a(s ; \mathbf{q}), b(s ; \mathbf{q})$ and $c(-s ; \mathbf{q})$ are (Hurwitz) stable for all $\mathbf{q} \in Q$ and their coefficients are continuous nonlinear functions over $Q$. In order to avoid degeneracies, we assume

$$
\begin{gathered}
a_{n-h}(\mathbf{q})>0 \quad b_{p}(\mathbf{q})>0, \quad \forall \mathbf{q} \in Q \\
\left\{c_{m}(\mathbf{q})>0 \forall \mathbf{q} \in Q\right\} \vee\left\{c_{m}(\mathbf{q})<0 \forall \mathbf{q} \in Q\right\} .
\end{gathered}
$$

Considering the control system depicted in Fig. 1, we search for a feedforward/feedback strategy in order to obtain a "robust" transition from a previous set-point value $y_{0}$ to a new one $y_{1}$. Without loss of generality in the following we will assume $y_{0}=0$. Obviously, the first requirement to be satisfied is the robust stability of the closed-loop over the uncertain domain $Q$. Moreover, this transition has to satisfy an overshoot and an undershoot limitation, an amplitude constraint on the control variable $u(t)$ and has to minimize the (worst-case) settling time. In other words, the above problem can be stated as follows: determine a command function $r(t)$ and a controller $C(s)$ such that

1) the closed-loop system is stable for all $\mathbf{q} \in Q$;

2) $\lim _{t \rightarrow \infty} y(t)=y_{1}$ for all $\mathbf{q} \in Q$ (steady-state condition);

3) the overshoot in response to $r(t)$ is bounded by a given $\bar{O}$ for all $\mathbf{q} \in Q$

4) the undershoot in response to $r(t)$ is bounded by a given $\bar{U}$ for all $\mathbf{q} \in Q$ 


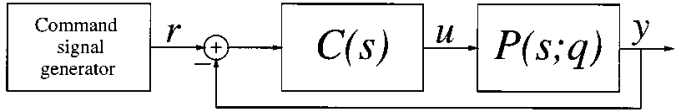

Fig. 1. The control scheme.

5) the absolute value of the manipulative input $u(t)$ is bounded by a given $u_{\text {sat }}$ for all $\mathbf{q} \in Q$;

6) it is minimized the worst-case settling time.

Searching for the true global solution of the above problem is extremely difficult, so that in the following we search for a practicable suboptimal but effective solution using "transition" polynomials [1] and a stable dynamic inversion procedure.

\section{ThE NeW InVERSION-BASED CONTROL ARCHITECTURE}

Introduce the "nominal" parameter vector $\mathbf{q}^{0}:=\operatorname{mid}(Q)$, i.e., the midpoint center of the box $Q$. Then, the following biproper parameterized controller, based on nominal stable pole-zero cancellations, is chosen:

$$
\begin{array}{ll}
C(s ; \alpha):=\operatorname{sign}\left(c\left(0 ; \mathbf{q}^{0}\right)\right) \alpha \frac{a\left(s ; \mathbf{q}^{0}\right)}{s b\left(s ; \mathbf{q}^{0}\right) d(s)}, & \text { if } h=0 \\
C(s ; \alpha):=\operatorname{sign}\left(c\left(0 ; \mathbf{q}^{0}\right)\right) \alpha \frac{a\left(s ; \mathbf{q}^{0}\right)}{b\left(s ; \mathbf{q}^{0}\right) d(s)}, & \text { if } h=1
\end{array}
$$

where $\alpha \in \mathbb{R}^{+}$and $d(s)$ is a user-chosen monic polynomial of degree $n-p-1$. In any case ( $h=0$ or $h=1)$, the closed-loop characteristic polynomial is

$$
s b\left(s ; \mathbf{q}^{0}\right) d(s) a(s ; \mathbf{q})+\operatorname{sign}\left(c\left(0 ; \mathbf{q}^{0}\right)\right) \alpha a\left(s ; \mathbf{q}^{0}\right) c(s ; \mathbf{q}) b(s ; \mathbf{q})
$$

which has degree $2 n$ if $h=0$ and $2 n-1$ if $h=1$.

Proposition 1: Assume that the monic polynomial $d(s)$ is Hurwitz stable. Then, there exists a sufficiently small $\alpha \in \mathbb{R}^{+}$such that the closed-loop system is stable for all $\mathbf{q} \in Q$.

Proof: Omitted for brevity. It is a straightforward consequence of a root locus technique.

The ideal output functions are designed using "transition" polynomials, which determine a smooth transition between 0 and $y_{1}$, to be accomplished without undershooting and overshooting in the time interval $[0, \tau]$. Specifically, we consider over the domain $[0, \tau]$ a polynomial function $y(t)$ of order $2 v+1$ :

$$
y(t)=c_{2 v+1} t^{2 v+1}+c_{2 v} t^{2 v}+\cdots+c_{1} t+c_{0}
$$

where the $2 v+2$ coefficients are determined by solving the following parameterized system:

$$
\begin{cases}y(0)=0 ; & y(\tau)=y_{1} \\ y^{(1)}(0)=0 ; & y^{(1)}(\tau)=0 \\ \vdots & \\ y^{(v)}(0)=0 ; & y^{(v)}(\tau)=0 .\end{cases}
$$

The above algebraic system always admits a unique solution which yields to the following closed-form expression of the desired output function [1]:

$$
\begin{array}{r}
y(t ; \tau):=y_{1} \frac{(2 v+1) !}{v ! \tau^{2 v+1}} \sum_{i=0}^{v} \frac{(-1)^{v-i}}{i !(v-i) !(2 v-i+1)} \tau^{i} t^{2 v-i+1}, \\
t \in[0, \tau] .
\end{array}
$$

The order of the polynomial is selected to obtain a function $y(t ; \tau)$ belonging to $C^{v}$ over $(-\infty,+\infty)$. Outside the interval $[0, \tau]$ the function $y(t ; \tau)$ is equal to 0 for $t<0$ and equal to $y_{1}$ for $t>\tau$.
The parameterized family of ideal output transfers defined by (5) being built over a polynomial basis is computationally simple and suitable to be implemented in a variety of digital control applications (see [6] exploiting transition polynomials for the point-to-point motion planning of vibratory systems)

\section{A. Command Input Synthesis via Stable Inversion}

The devised function $y(t ; \tau)$ of formula (5) is the ideal plant output to be obtained by injecting an appropriate command signal $r(t)$ into the control scheme of Fig. 1. A natural choice is to compute the command signal by means of a dynamic inversion technique. Specifically, we consider the nominal plant transfer function so that the overall dynamics from $r$ to $y$ are simplified by exact stable pole-zero cancellations between the controller and the plant. The nominal open-loop transfer function is

$$
L(s ; \alpha):=\operatorname{sign}\left(c\left(0 ; \mathbf{q}^{0}\right)\right) \alpha \frac{\left(c\left(s ; \mathbf{q}^{0}\right)\right)}{s d(s)}
$$

and the corresponding transfer function from $r$ to $y$ is given by

$$
T_{y r}(s ; \alpha):=\frac{\operatorname{sign}\left(c\left(0 ; \mathbf{q}^{0}\right)\right) \alpha c\left(s ; \mathbf{q}^{0}\right)}{s d(s)+\operatorname{sign}\left(c\left(0 ; \mathbf{q}^{0}\right)\right) \alpha c\left(s ; \mathbf{q}^{0}\right)} .
$$

The order of $T_{y r}(s ; \alpha)$ is $n-p$ and its relative order is $\rho$. Denote with $Y(s ; \tau)$ the Laplace transform of $y(t ; \tau)$ and, performing the inverse Laplace transformation, compute

$$
r_{u}(t ; \alpha, \tau):=\mathcal{L}^{-1}\left[T_{y r}^{-1}(s ; \alpha) Y(s ; \tau)\right] .
$$

First, we note that, due to the relative degree of $T_{y r}(s ; \alpha), r_{u}(t ; \alpha, \tau)$ is continuous over $(-\infty,+\infty)$ only if $v \geq \rho$, i.e., the desired output $y(t ; \tau)$ has to belong to $C^{\rho}$ at least. Second, we observe that $r_{u}(t ; \alpha, \tau)$ is the command signal computed with the standard dynamic inversion technique, i.e., the method that consider the overall system at the equilibrium for $t=0$. Unfortunately, due to the unstable zero dynamics, $r_{u}(t ; \alpha, \tau)$ is unbounded over $[0, \infty)$, so that it is useless for command signal purposes. As a consequence, it is necessary to introduce the following problem.

Stable Inversion Problem: Assume that the nominal closed-loop system is at the equilibrium for $t=-\infty$. Determine a bounded command signal defined over $(-\infty,+\infty)$ such that the corresponding output is exactly given by $y(t ; \tau)$.

In the following, we give an explicit constructive solution to the above problem. For notational simplicity we assume that all the zeros of $c\left(s ; \mathbf{q}^{0}\right)$ are distinct. Hence, polynomial $c\left(s ; \mathbf{q}^{0}\right)$ can be factorized according to:

$$
\begin{aligned}
c\left(s ; \mathbf{q}^{0}\right)=c_{z}\left(s-z_{1}\right) \cdots\left(s-z_{l}\right)\left(s^{2}-2 \delta_{1} \omega_{1} s+\omega_{1}^{2}\right) \\
\cdots\left(s^{2}-2 \delta_{h} \omega_{h} s+\omega_{h}^{2}\right)
\end{aligned}
$$

where $z_{i} \in \mathbb{R}^{+}, i=1, \ldots, l$ and $\omega_{j} \in \mathbb{R}^{+}, \delta_{j} \in(0,1), j=1, \ldots, h$. Define the command function $r(t ; \alpha, \tau)$ as follows:

$$
\begin{cases}r(t ; \alpha, \tau):=r_{u}(t ; \alpha, \tau)+r_{c}(t ; \alpha, \tau), & \text { for } t \leq \tau \\ r(t ; \alpha, \tau):=y_{1}, & \text { for } t>\tau\end{cases}
$$

where

$$
\begin{aligned}
r_{c}(t ; \alpha, \tau):= & k_{1} e^{z_{1}\left(t-t_{1}\right)}+\cdots+k_{l} e^{z_{l}\left(t-t_{l}\right)} \\
& +k_{l+1} e^{\delta_{1} \omega_{1}\left(t-t_{l+1}\right)} \\
& \times \sin \left(\omega_{1} \sqrt{1-\delta_{1}^{2}}\left(t-t_{l+1}\right)+\phi_{1}\right) \\
& +\cdots+k_{l+h} e^{\delta_{h} \omega_{h}\left(t-t_{l+h}\right)} \\
& \times \sin \left(\omega_{h} \sqrt{1-\delta_{h}^{2}}\left(t-t_{l+h}\right)+\phi_{h}\right) .
\end{aligned}
$$


Theorem 1: There exist $t_{i} \in \mathbb{R}, k_{i} \in\{-1,0,+1\}, i=1, \ldots, l+h$ and $\phi_{j} \in[0, \pi), j=1, \ldots, h$ such that the function $r(t ; \alpha, \tau)$ defined in (10) and (11) is the unique solution to the stable inversion problem.

Proof: A constructive proof of Theorem 1 is briefly sketched. The coefficients $t_{i}, k_{i}$ and $\phi_{i}$ appearing in (11) can be determined by solving the following equation system (note that $m=l+2 h$ ):

$$
\left\{\begin{array}{l}
r_{c}(\tau ; \alpha, \tau)=y_{1}-r_{u}\left(\tau^{+} ; \alpha, \tau\right) \\
D r_{c}(\tau ; \alpha, \tau)=-D r_{u}\left(\tau^{+} ; \alpha, \tau\right) \\
\vdots \\
D^{m-1} r_{c}(\tau ; \alpha, \tau)=-D^{m-1} r_{u}\left(\tau^{+} ; \alpha, \tau\right)
\end{array}\right.
$$

where $(i=0,1, \ldots, m-1)$

$$
D^{i} r_{u}\left(\tau^{+} ; \alpha, \tau\right):=\lim _{t \rightarrow \tau^{+}} D^{i} r_{u}(t ; \alpha, \tau)
$$

As it will be clear in the subsequent passages [see (19)], the above system (12) imposes an exact cancellation over $(\tau,+\infty)$ of the unstable modes appearing in $r_{u}(t ; \alpha, \tau)$ with those of $r_{c}(t ; \alpha, \tau)$. For brevity, we expose the case of $m=l=2$ (see the example in Section V).

From (12), we deduce

$$
\left\{\begin{array}{l}
k_{1} e^{z_{1}\left(\tau-t_{1}\right)}+k_{2} e^{z_{2}\left(\tau-t_{2}\right)}=y_{1}-r_{u}\left(\tau^{+} ; \alpha, \tau\right) \\
k_{1} z_{1} e^{z_{1}\left(\tau-t_{1}\right)}+k_{2} z_{2} e^{z_{2}\left(\tau-t_{2}\right)}=-D r_{u}\left(\tau^{+} ; \alpha, \tau\right) .
\end{array}\right.
$$

Hence, defining

$$
\begin{aligned}
& \eta_{1}:=z_{2}\left[y_{1}-r_{u}(\tau ; \alpha, \tau)\right]+D r_{u}\left(\tau^{+} ; \alpha, \tau\right) \\
& \eta_{2}:=-z_{1}\left[y_{1}-r_{u}(\tau ; \alpha, \tau)\right]-D r_{u}\left(\tau^{+} ; \alpha, \tau\right)
\end{aligned}
$$

it follows that:

$$
\begin{aligned}
& k_{1} e^{z_{1}\left(\tau-t_{1}\right)}=\eta_{1}\left(z_{2}-z_{1}\right)^{-1} \\
& k_{2} e^{z_{2}\left(\tau-t_{2}\right)}=\eta_{2}\left(z_{2}-z_{1}\right)^{-1} .
\end{aligned}
$$

The coefficients $k_{1}$ and $k_{2}$ are computed according to

$$
k_{1}=\operatorname{sign}\left[\eta_{1}\left(z_{2}-z_{1}\right)^{-1}\right], \quad k_{2}=\operatorname{sign}\left[\eta_{2}\left(z_{2}-z_{1}\right)^{-1}\right] .
$$

Therefore, from (16) and using (17), we obtain

$$
\begin{aligned}
& t_{1}=\tau-z_{1}^{-1} \ln \left[\eta_{1} k_{1}\left(z_{2}-z_{1}\right)^{-1}\right] \\
& t_{2}=\tau-z_{2}^{-1} \ln \left[\eta_{2} k_{2}\left(z_{2}-z_{1}\right)^{-1}\right] .
\end{aligned}
$$

It is evident from the above passages that the found solution (17)-(18) corresponds to a unique function $r_{c}(t ; \alpha, \tau)$ satisfying system (14). This fact also holds in full generality $(l, h \in \mathbb{N})$.

In the following, consider the nominal closed-loop system at the equilibrium for $t=-\infty$. Applying the command signal given by (11), defined on $(-\infty,+\infty)$, we evidently have that the output signal is zero over $(-\infty,+\infty)$, for any choice of the involved coefficients $t_{i}, k_{i}$ and $\phi_{j}$. Hence, by virtue of linear superposition, applying $r_{u}(t ; \alpha, \tau)+$ $r_{c}(t ; \alpha, \tau)$ as a command signal, defined over $(-\infty,+\infty)$, the output is given by $y(t ; \tau)$.

Now it is shown that, for the special choice of coefficients $t_{i}, k_{i}$ and $\phi_{j}$ satisfying system (12), also the command signal defined in (10) gives the signal $y(t ; \tau)$ as corresponding output. Indeed, the nominal closed-loop system is at the equilibrium for $t>\tau$.
Denote the transfer function $T_{y r}(s ; \alpha)$ as

$$
T_{y r}(s ; \alpha)=\frac{\mu_{m} s^{m}+\cdots+\mu_{1} s+1}{\lambda_{n-p} s^{n-p}+\cdots+\lambda_{1} s+1}
$$

so that the differential equation describing the nominal closed-loop dynamics is

$$
\lambda_{n-p} D^{n-p} y+\cdots+\lambda_{1} D y+y=\mu_{m} D^{m} r+\cdots+\mu_{1} D r+r .
$$

This equation is satisfied over $(-\infty,+\infty)$ by functions $r_{u}(t ; \alpha, \tau)+$ $r_{c}(t ; \alpha, \tau)$ and $y(t ; \tau)$. Hence, for $t>\tau$ it follows that

$$
\begin{aligned}
& \mu_{m} D^{m}\left[r_{u}(t ; \alpha, \tau)+r_{c}(t ; \alpha, \tau)\right] \\
& \quad+\cdots+\mu_{1} D\left[r_{u}(t ; \alpha, \tau)+r_{c}(t ; \alpha, \tau)\right] \\
& \quad+r_{u}(t ; \alpha, \tau)+r_{c}(t ; \alpha, \tau)=y_{1} .
\end{aligned}
$$

Taking into account that $r_{c}(t ; \alpha, \tau) \in C^{\infty}$, from (12) we have

$$
\left\{\begin{array}{l}
r_{u}\left(\tau^{+} ; \alpha, \tau\right)+r_{c}\left(\tau^{+} ; \alpha, \tau\right)=y_{1} \\
D\left[r_{u}\left(\tau^{+} ; \alpha, \tau\right)+r_{c}\left(\tau^{+} ; \alpha, \tau\right)\right]=0 \\
\vdots \\
D^{m-1}\left[r_{u}\left(\tau^{+} ; \alpha, \tau\right)+r_{c}\left(\tau^{+} ; \alpha, \tau\right)\right]=0
\end{array}\right.
$$

where the definition of $D^{i}\left[r_{u}\left(\tau^{+} ; \alpha, \tau\right)+r_{c}\left(\tau^{+} ; \alpha, \tau\right)\right]$ is analogous to that in (13). Consequently, by virtue of the uniqueness result of ordinary differential equations,

$$
r_{u}(t ; \alpha, \tau)+r_{c}(t ; \alpha, \tau)=y_{1} \quad \forall t>\tau .
$$

Evidently, $r_{u}(t ; \alpha, \tau)$ is bounded over $(-\infty, \tau]$ and, considering that all the roots of $c\left(s ; \mathbf{q}^{0}\right)$ have positive real parts, also $r_{c}(t ; \alpha, \tau)$ is bounded over $(-\infty, \tau]$. Therefore, for the special performed choice of the coefficients, $r_{u}(t ; \alpha, \tau)+r_{c}(t ; \alpha, \tau)$ is bounded over $(-\infty,+\infty)$ and coincides with the function $r(t ; \alpha, \tau)$ defined in (10).

In order to practically use the synthesized function (10) it is necessary to truncate $r(t ; \alpha, \tau)$ resulting in an approximate generation of the desired output $y(t ; \tau)$. This can be done with arbitrarily precision given any small parameter $\varepsilon_{0}>0$. Indeed, compute

$$
t_{o c}:=\max \left\{t_{c}:\left|r_{c}(t ; \alpha, \tau)\right| \leq \varepsilon_{0} \quad \forall t \in\left(-\infty, t_{c}\right]\right\}
$$

and define

$$
t_{0}:=\min \left\{0, t_{o c}\right\}
$$

Hence, the approximate command signal to be used is

$$
r_{a}(t ; \alpha, \tau):= \begin{cases}0, & \text { for } t<t_{0} \\ r(t ; \alpha, \tau), & \text { for } t \geq t_{0} .\end{cases}
$$

Note that $t_{0}$ depends on both $\alpha$ and $\tau$. Moreover, it can happen that $t_{0}<0$, resulting in the so-called "preaction control" [7].

\section{DESIGN Procedure}

In Section III, we have determined a controller $C(s ; \alpha)$ and a command signal $r_{a}(t ; \alpha, \tau)$ that depend on the free positive parameters $\alpha$ and $\tau$. The design procedure to solve the set-point constrained regulation problem posed in Section II can then be outlined as follows:

- choose the monic polynomial $d(s)$ ensuring robust closed-loop stability for at least one positive value of $\alpha$;

- determine the optimal parameters $\alpha^{*}$ and $\tau^{*}$ that minimize the worst-case settling time subject to all the required constraints. 

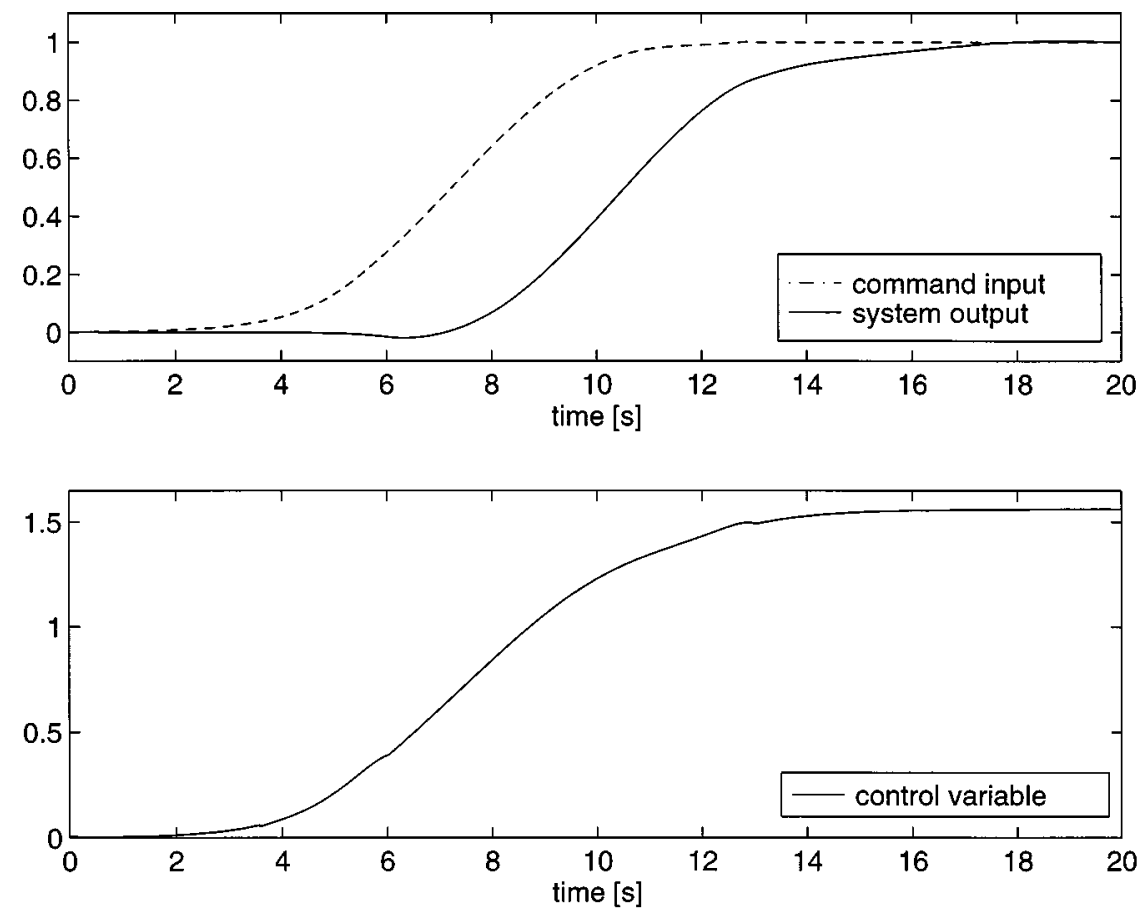

Fig. 2. The optimal command input, worst-case system output and corresponding control signal for the worked example.
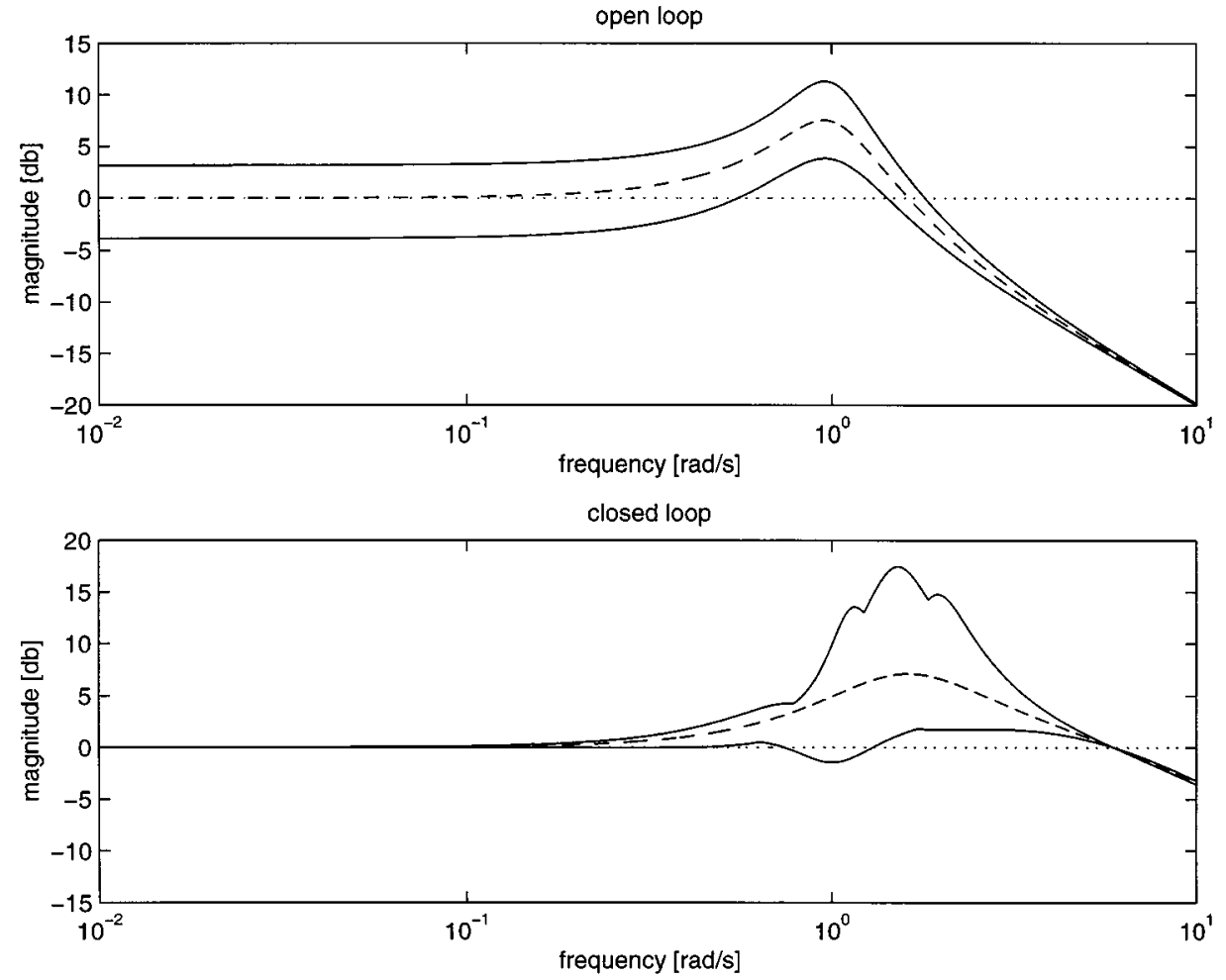

Fig. 3. Envelope of the Bode plots over the uncertain parameters box $\mathcal{Q}$ (solid) and Bode plot of the nominal case (dashed) for the plant and for the closed-loop system of the example.

Step 1 can be simply accomplished by choosing a (Hurwitz) stable $d(s)$. Indeed, Proposition 1 assures that robust stability is achieved for a sufficiently small $\alpha$. It is worth noting that $d(s)$ may be not stable; for example, see the design exposed in Section V. In any case, the control engineer may select the polynomial $d(s)$ on the grounds of bandwidth considerations, root locus reasoning, physical judgement, etc. Robust stability can be taken into account by using the following result that can be derived from Lienard and Chipard's criterion and Orlando's formula (cf. [8]).

Proposition 2: The closed-loop system is stable for all $\mathbf{q} \in \mathcal{Q}$ if and only if

$$
\begin{aligned}
S_{1}(\alpha, \mathbf{q})>0 & S_{2}(\alpha, \mathbf{q})>0, \quad \forall \mathbf{q} \in \mathcal{Q} \\
S_{3}\left(\alpha, \mathbf{q}^{0}\right)>0 & S_{4}\left(\alpha, \mathbf{q}^{0}\right)>0, \ldots, S_{n_{c}}\left(\alpha, \mathbf{q}^{0}\right)>0
\end{aligned}
$$




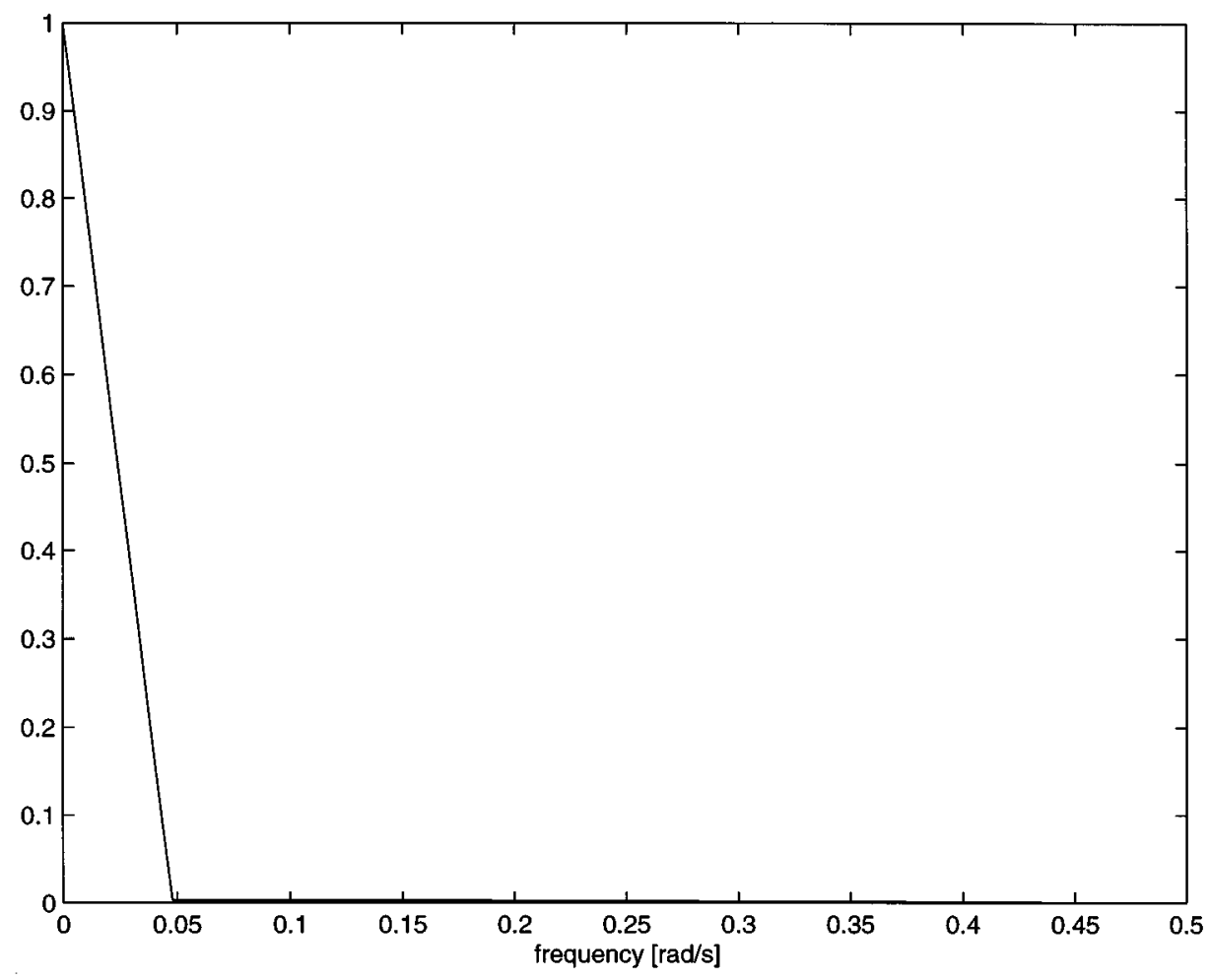

Fig. 4. Normalized power spectrum of the command signal of the example.

where $n_{c}$ is the degree of the characteristic polynomial (4) and functions $S_{i}(\alpha, \mathbf{q}) i=1, \ldots, n_{c}$ are related coefficients or Hurwitz determinants as explained in [8].

Define $y(t ; \alpha, \tau, \mathbf{q})$ and $u(t ; \alpha, \tau, \mathbf{q})$ the output and control signals respectively when the command function is $r_{a}(t ; \alpha, \tau)$. The associated settling time can be pertinently defined as

$$
\begin{aligned}
& t_{s}(\alpha, \tau, \mathbf{q}) \\
& :=\left|t_{0}\right|+\min \left\{s \in \mathbb{R}^{+}:\left|y(t ; \alpha, \tau, \mathbf{q})-y_{1}\right| \leq 0.02 y_{1} \forall t \geq s\right\} .
\end{aligned}
$$

Consequently, the worst-case settling time is

$$
t_{w c s}(\alpha, \tau):=\max _{\mathbf{q} \in \mathcal{Q}} t_{s}(\alpha, \tau, \mathbf{q})
$$

Without conservativeness, the optimal choice of $\alpha$ and $\tau$ entails solving the following semi-infinite optimization problem

$$
\min _{\alpha, \tau \in \mathbb{R}^{+}} t_{w c s}(\alpha, \tau)
$$

subject to

$$
\begin{aligned}
S_{1}(\alpha, \mathbf{q}) & \geq \epsilon \quad S_{2}(\alpha, \mathbf{q}) \geq \epsilon, \quad \forall \mathbf{q} \in \mathcal{Q} \\
S_{3}\left(\alpha, \mathbf{q}^{0}\right) & \geq \epsilon \quad S_{4}\left(\alpha, \mathbf{q}^{0}\right) \geq \epsilon, \ldots, S_{n_{c}}\left(\alpha, \mathbf{q}^{0}\right) \geq \epsilon \\
y(t ; \alpha, \tau, \mathbf{q}) & \leq(1+0.01 \bar{O}) y_{1}, \quad \forall t \geq 0 \quad \forall \mathbf{q} \in \mathcal{Q} \\
y(t ; \alpha, \tau, \mathbf{q}) & \geq-0.01 \bar{U} y_{1}, \quad \forall t \geq 0 \forall \mathbf{q} \in \mathcal{Q} \\
|u(t ; \alpha, \tau, \mathbf{q})| & \leq u_{\text {sat }}, \quad \forall t \geq 0, \quad \forall \mathbf{q} \in \mathcal{Q}
\end{aligned}
$$

where $\varepsilon$ is a sufficiently small threshold parameter.

Remark: Note that the settling time definition incorporates the preaction time $\left|t_{0}\right|$ even though during the interval $\left(t_{0}, 0\right)$ the output signal is almost identically zero. This appears technically sound because during $\left(t_{0}, 0\right)$ the overall system is out of equilibrium.
Solving problem (24) means to find solution to the complete setpoint regulation problem within the framework of the proposed inversion-based control architecture. Indeed all the control requirements are incorporated as inequality constraints of (24) with the exception of the steady-state regulation condition that is guaranteed through robust stability by virtue of the controller structure (the internal model principle is satisfied). The main result of this section is the following.

Theorem 2: For any given overshoot limit $\bar{O} \in \mathbb{R}^{+}$and any undershoot limit $\bar{U} \in \mathbb{R}^{+}$, optimization problem (24) has a solution if

$$
\begin{aligned}
& u_{\text {sat }}>\left(\min _{\mathbf{q} \in \mathcal{Q}}|P(0, \mathbf{q})|\right)^{-1}\left|y_{1}\right|, \quad \text { when } h=0 \\
& u_{\text {sat }}>0, \quad \text { when } h=1 .
\end{aligned}
$$

Proof: Omitted for brevity. It is an extension of a proof presented in [2].

Problem (24) is a nonlinear semi-infinite optimization problem for which an approximate solution can be obtained by relaxing the semiinfinite constraints. For example, the box $\mathcal{Q}$ can be substituted with its vertexes and a genetic algorithm [9] can be adopted for estimating $\alpha^{*}$ and $\tau^{*}$. This approach requires, as explained in [2], an algorithmic postprocessing to ensure the feasibility of the solution. A more effective but effortful, approach could be using the genetic/interval algorithm of Lo Bianco and Piazzi [10].

\section{A WORKED EXAMPLE}

As an illustrative example, we consider a plant with $h=0$ :

$$
P(s ; \mathbf{q})=\frac{\left(s-q_{1}\right)\left(s-q_{2}\right)}{\left(s^{2}+2 q_{3} s+1\right)(s+2)}
$$

where $\mathbf{q}=\left[q_{1}, q_{2}, q_{3}\right] \in Q=[0.8,1.2] \times[1.6,2.4] \times[0.5,0.7]$. We also have fixed $y_{1}=1, \bar{O}=5 \%, \bar{U}=3 \%$ and $u_{\text {sat }}=2$. Following the methodology described in this note, we set $a\left(s ; \mathbf{q}^{0}\right)=$ $\left(s^{2}+2 q_{3}^{0} s+1\right)(s+2)=\left(s^{2}+1.2 s+1\right)(s+2), b\left(s ; \mathbf{q}^{0}\right)=1$, and 
$c\left(s ; \mathbf{q}^{0}\right)=\left(s-q_{1}^{0}\right)\left(s-q_{2}^{0}\right)=(s-1)(s-2)$. Hence, the following controller results (note that $m=2$ ):

$$
C(s ; \alpha)=\alpha \frac{\left(s^{2}+1.2 s+1\right)(s+2)}{s d(s)} .
$$

We selected $d(s)=s^{2}+35$, so that all the poles of $C(s ; \alpha)$ are purely imaginary. In order to have $r(t ; \alpha, \tau) \in C^{0}$, a third-order transition polynomial ( $v=1$, note that the plant relative order is $\rho=1$ ) has been chosen as output function, so that we have

$$
y(t ; \tau)=-\frac{2}{\tau^{3}} t^{3}+\frac{3}{\tau^{2}} t^{2} \quad t \in[0, \tau]
$$

The optimal values of $\alpha$ and $\tau$ have been determined by means of a genetic algorithm [9]. The resulting values are $\alpha^{*}=7.57$ and $\tau^{*}=$ $6.99 \mathrm{~s}$, which results in an optimal worst-case settling time $t_{w c s}^{*}$ equal to $16.44 \mathrm{~s}$, with preaction time $t_{0}^{*}=-6.2 \mathrm{~s}\left(\varepsilon_{0}=10^{-3}\right)$. The command input $r_{a}\left(t ; \alpha^{*}, \tau^{*}\right)$, the worst-case output (which occurs when $\mathbf{q}=$ $[0.8,1.6,0.5])$ and the corresponding control variable are reported in Fig. 2. For technical convenience, in all the plots the zero time has been shifted to $t_{0}^{*}$.

To better evaluate how the new proposed approach works, in Fig. 3 we report the envelope of the Bode plots over the uncertain parameters box $\mathcal{Q}$, both of the plant (open-loop) and of the closed-loop system. Then, in Fig. 4 the normalized power spectrum of the command signal is shown. It can be noted that for the range of frequencies of the command signal, the presence of the feedback controller almost completely cancels the effects of the plant uncertainties, therefore greatly increasing the effectiveness of the use of the dynamic inversion.

\section{CONCLUSION}

In this note, we have proposed a new inversion-based control architecture for the set-point constrained regulation of nonminimum-phase scalar systems subject to parametric uncertainties. This new control scheme, which can be regarded as a radical generalization of the classic two-degrees-of-freedom configuration (the filter is indeed substituted by the command signal generator, see Fig. 1), permits obtaining high performances as exemplified in Section V. An extension to multivariable plants is possible and is currently investigated by the authors.

\section{REFERENCES}

[1] A. Piazzi and A. Visioli, "Optimal noncausal set-point regulation of scalar systems," Automatica, vol. 37, no. 1, pp. 121-127, Jan. 2001

[2] - "Robust set-point constrained regulation via dynamic inversion," Int. J. Robust Nonlinear Control, vol. 11, no. 1, pp. 1-22, Jan. 2001.

[3] S. Devasia, D. Chen, and B. Paden, "Nonlinear inversion-based output tracking," IEEE Trans. Automat. Contr., vol. 41, pp. 930-942, July 1996.

[4] L. R. Hunt and G. Meyer, "Stable inversion for nonlinear systems," $A u$ tomatica, vol. 33, no. 8, pp. 1549-1554, 1997.

[5] S. Devasia, "Robust inversion-based feedforward controllers for output tracking under plant uncertainty," in Proc. Amer. Control Conf., Chicago, IL, June 2000, pp. 497-502.

[6] A. Piazzi and A. Visioli, "Minimum-time system inversion based motion planning for residual vibration reduction," IEEE Trans. Mechatron., vol. 5, pp. 12-22, Mar. 2000.

[7] G. Marro, "Multivariable regulation in geometric terms: Old and new results," in Colloquium on Automatic Control, C. Bonivento, G. Marro, and R. Zanasi, Eds. New York: Springer-Verlag, 1996, pp. 77-138.

[8] A. Piazzi and A. Visioli, "Noncausal robust set-point regulation of nonminimum-phase scalar systems," in Proc. 39th IEEE Conf. Decision Control, Sydney, Australia, Dec. 2000, pp. 4098-4103.

[9] C. Houck, J. Joines, and M. Kay, "A genetic algorithm for function optimization: a Matlab implementation,”, NCSU-IE TR 95-09, 1995.

[10] C. Guarino Lo Bianco and A. Piazzi, "A hybrid algorithm for infinitely constrained optimization," Int. J. Syst. Sci., vol. 32, no. 1, pp. 91-102, Jan. 2001

\section{New Iterative Algorithm for Algebraic Riccati Equation Related to $H_{\infty}$ Control Problem of Singularly Perturbed Systems}

\author{
Hiroaki Mukaidani, Hua Xu, and Koichi Mizukami
}

\begin{abstract}
In this note, we present the solution to the algebraic Riccati equation (ARE) with indefinite sign quadratic term related to the $\boldsymbol{H}_{\infty}$ control problem for singularly perturbed system by means of a Kleinman's type algorithm. The resulting algorithm is very efficient from the numerical point of view because the ARE is solvable even if the quadratic term has an indefinite sign. Moreover, the resulting iterative algorithm is quadratically convergent. We also present a new algorithm for solving the generalized algebraic Lyapunov equation (GALE) on the basis of the fixed point algorithm.
\end{abstract}

Index Terms-Fixed point algorithm, $H_{\infty}$ control, Kleinman algorithm, singularly perturbed systems.

\section{INTRODUCTION}

$H_{\infty}$ control problems for linear singularly perturbed systems were considered in many papers [1]-[9]. In particular, a great deal of studies on the composite controller design for singularly perturbed systems in $H_{\infty}$ sense have been made [2], [3], [6], [8].

In order to obtain the optimal controller, we must solve the algebraic Riccati equation (ARE). Various reliable approaches to the theory of the ARE have been well documented in many literatures (see e.g., [11]-[14]). These methods consist of the invariant subspace approach which is based on the Hamiltonian matrix [11], [12] and the general matrix pencil technique which is based on the extended Hamiltonian pencil [13], [14] (in particular, the reference [14] is the most complete reference to date dealing with ARE by means of the matrix pencils). However, such approaches are not adequate to the singularly perturbed systems because of high dimension and numerical stiffness [10].

The recursive algorithm for the solution of ARE of singularly perturbed systems have been developed in many literatures (see, e.g., [15]). From a practical point of view, it has been shown that the recursive algorithm is very effective to solve the ARE when the system matrices are functions of a small perturbation parameter $\varepsilon$. However, the recursive algorithm converge only to the approximation solution. Moreover, such an algorithm is the linear convergence. On the other hand, the exact slow-fast decomposition method for solving the singularly perturbed systems has been proposed (see, for example, [7] and the references therein). However, in order to obtain the exact solution, ones need the same workspace compared with with the full-order ARE for calculating the inverse matrix.

In this paper, we study the numerical solution to the ARE with indefinite sign quadratic term related to the $H_{\infty}$ control problem of singularly perturbed systems. The objective of this paper is to extend the convergence result of [17] to the ARE with indefinite sign quadratic term. Our new idea is to set the initial condition to the solutions of the reduced-order ARE. Because of such a choice, we can prove that our iterative algorithm converges to a unique solution of the ARE

Manuscript received January 4, 2000; revised August 29, 2000 and February 7, 2001. Recommended by Associate Editor C. Oara.

H. Mukaidani is with the Faculty of Information Sciences, Hiroshima City University, Hiroshima, Japan (e-mail: mukaida@im.hiroshima-cu.ac.jp).

$\mathrm{H}$. Xu is with the Graduate School of Business Sciences, The University of Tsukuba, Tokyo, Japan.

K. Mizukami is with the Faculty of Engineering, Hiroshima Kokusai Gakuin University, Hiroshima, Japan.

Publisher Item Identifier S 0018-9286(01)09544-7. 